\title{
Erratum to: Comparison of acyclovir and famciclovir for the treatment of Bell's palsy
}

\author{
Ho Joong Kim ${ }^{1}$ - Sang Hoon Kim ${ }^{1}$ Junyang Jung ${ }^{2}$ - Sung Su Kim ${ }^{3}$ • \\ Jae Yong Byun ${ }^{1} \cdot$ Moon Suh Park ${ }^{1} \cdot$ Seung Geun Yeo ${ }^{1}$
}

Published online: 8 August 2016

(C) Springer-Verlag Berlin Heidelberg 2016

\section{Erratum to: Eur Arch Otorhinolaryngol \\ DOI 10.1007/s00405-016-3927-3}

In Table 4, the value for Famciclovir and Valaciclovir were incorrect in the original publication. The value for Famciclovir should be $750 \mathrm{mg}$ instead of $250 \mathrm{mg}$, and Valaciclovir should be $1000 \mathrm{mg}$ instead of $100 \mathrm{mg}$. Also, the last entry in the column "Antiviral dose" should be 7 days instead of 10 days. The corrected table is given below:

The online version of the original article can be found under doi:10.1007/s00405-016-3927-3.

Seung Geun Yeo

yeo2park@gmail.com

1 Department of Otohinolaryngology, H \& N Surgery, School of Medicine, Kyung Hee University, \#1 Hoegi-dong, Dongdaemun-gu, Seoul 130-702, Korea

2 Department of Anatomy, School of Medicine, Kyung Hee University, Seoul, Korea

3 Department of Biochemistry and Molecular Biology, Medical Science and Engineering Research Center for Bioreaction to Reactive Oxygen Species, BK-21, School of Medicine, Kyung Hee University, Seoul 130-701, Korea 


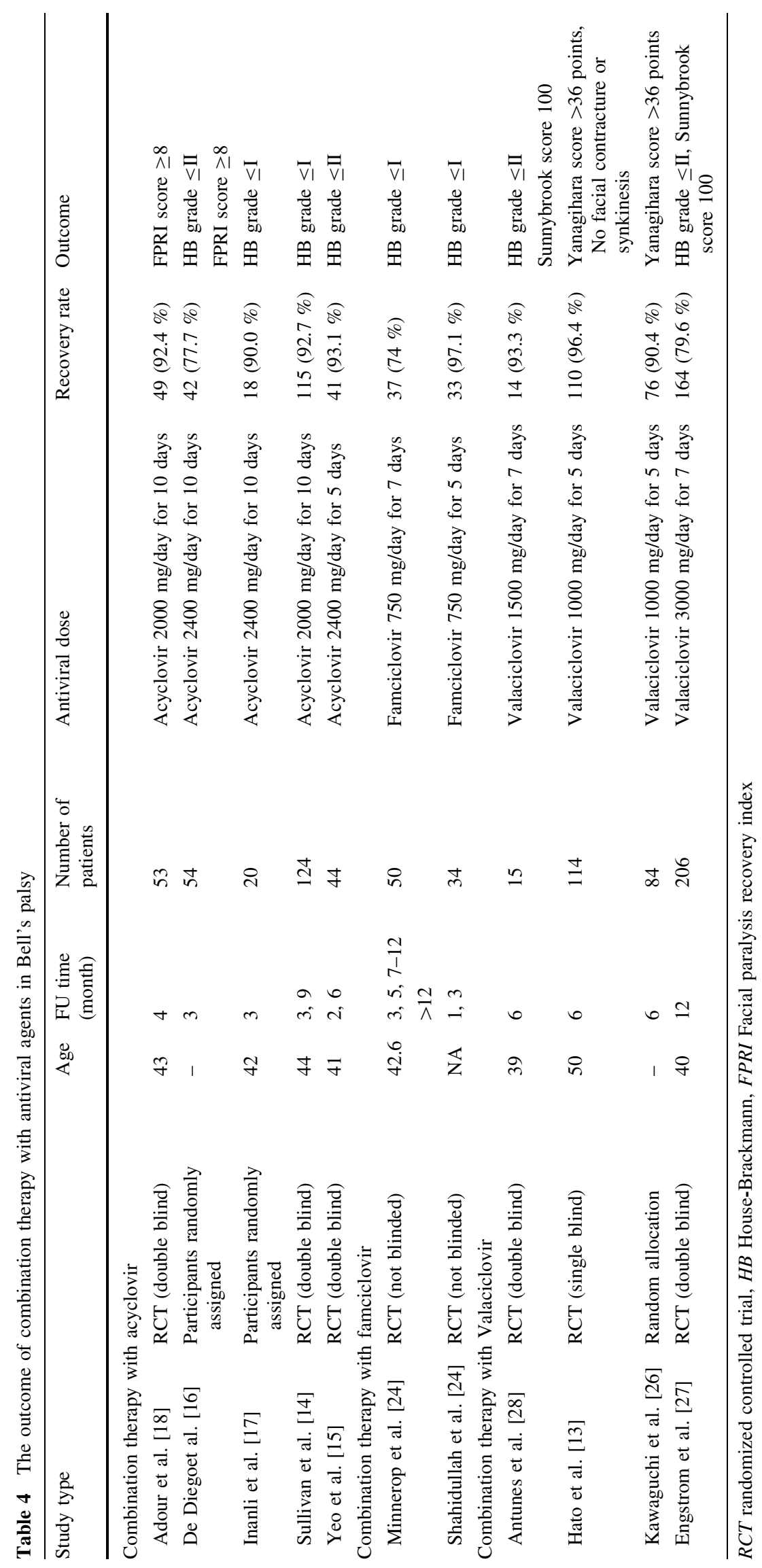

